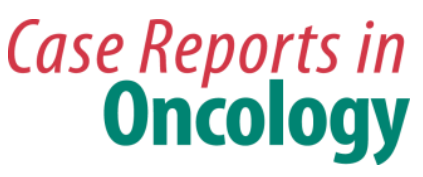

Case Rep Oncol 2016;9:415-421

DOI: $10.1159 / 00044771$

Publisned onine: August 10, 2016

(C) 2016 The Author(s)

Published by S. Karger AG, Basel

www.karger.com/cro

This article is licensed under the Creative Commons Attribution-NonCommercial 4.0 International License (CC BY-NC) (http://www.karger.com/Services/OpenAccessLicense).

Usage and distribution for commercial purposes requires written permission.

\title{
An Atypical Initial Presentation of Chronic Myeloid Leukemia with Central Nervous System and Lymph Node Blast Crises
}

\author{
Khadega A. Abuelgasim ${ }^{a} b \quad$ Saeed Alshieban ${ }^{c} \quad$ Nada A. Almubayi ${ }^{d}$ \\ Ayman Alhejazi $^{\mathrm{a}} \quad$ Abdulrahman R. Jazieh $^{\mathrm{a}}$ \\ ${ }^{a}$ Department of Oncology, King Abdulaziz Medical City, NGHA, Riyadh, Saudi Arabia; \\ ${ }^{b}$ King Abdulla International Medical Research Center, King Saud Bin Abdulaziz University \\ for Health Sciences, NGHA, Riyadh, Saudi Arabia; ${ }^{\mathrm{C}}$ Department of Pathology, King \\ Abdulaziz Medical City, NGHA, Riyadh, Saudi Arabia; ${ }^{d}$ King Saud Bin Abdulaziz University \\ for Health Sciences, Nursing School, Riyadh, Saudi Arabia
}

\section{Keywords}

Chronic myeloid leukemia · Blast crisis · Central nervous system · Lymph nodes

\begin{abstract}
We describe the case of a young man with therapy-naive chronic myeloid leukemia who did not initially have any peripheral blood or bone marrow excess blasts but presented with extramedullary myeloid blast crises involving the central nervous system and multiple lymph nodes. Conventional cytogenetic tests were positive for $\mathrm{t}(9 ; 22)(\mathrm{q} 34: \mathrm{q} 11)$ as well as for trisomy 8,14 and 21 and del(16q). The patient's peripheral blood and bone marrow were positive for the $B C R-A B L$ oncogene when analyzed by fluorescence in situ hybridization and polymerase chain reaction. He achieved good clinical, radiological, cytogenetic and molecular response to acute myeloid leukemia induction chemotherapy combined with 16 doses of triple intrathecal chemotherapy and oral dasatinib (second-generation tyrosine kinase inhibitor) treatment. Due to his poor general condition, he was treated with $24 \mathrm{~Gy}$ of whole-brain radiation therapy, as allogeneic stem cell transplantation was not feasible. Although extramedullary CNS blast crises are usually associated with a very poor outcome, our patient remains in complete cytogenetic and molecular remission, on single-agent dasatinib, 4 years after the
\end{abstract}


diagnosis with no current evidence of active extramedullary disease. This suggests that dasatinib has a role in controlling not only chronic-phase chronic myeloid leukemia, but also its CNS blast crisis.

\section{Background}

Chronic myeloid leukemia (CML) is a clonal myeloproliferative disorder involving increased proliferation of the granulocytes without loss of differentiation capacity. It is characterized by the presence of a reciprocal translocation between the long arms of chromosomes 9 and 22, $\mathrm{t}(9 ; 22)$ (q34:q11), resulting in the so-called Philadelphia chromosome; this translocation leads to the fusion of the break point cluster region (BCR) with the ABL gene, which forms an oncogene, the transcript of which is an oncoprotein with a tyrosine kinase function [1]. In the majority of cases, CML is diagnosed in the chronic phase; it is less frequently diagnosed in accelerated crises, and occasionally, its initial presentation is as acute leukemia (blast crisis). Approximately $80 \%$ of blast crises are of an acute myelogenous leukemia type, and the remaining are of an acute lymphoblastic leukemia type [2,3].

Extramedullary blast crises, which primarily affect the lymph nodes, are a known complication of CML, and they occur in up to $16 \%$ of CML cases [4]. Among these patients, approximately $70 \%$ have myeloid disease, and the remaining have lymphoid disease. In some cases, the blast crises can be of mixed myeloid and lymphoid origin [5, 6]. The majority of extramedullary involvement occurs in association with peripheral and/or bone marrow (BM) blastema; in rare cases, it may present as soft tissue leukemic infiltrate (chloroma, myeloblastoma or myeloid sarcoma) of different sites, such as lymph nodes, synovium or bone $[2-4,7-10]$.

CML patients rarely present with a CNS blast crisis, with CNS crisis usually being reported in patients treated with imatinib [7-14]. CNS blast crisis is likely due to the poor penetration of the drug through the blood-brain barrier; therefore, the CNS acts as a sanctuary site $[15,16]$.

Here, we describe the case of a young man who was diagnosed with CML when he presented with treatment-naive extramedullary myeloid blast crises involving the CNS and multiple lymph nodes, with no features of accelerated or blast crises in the peripheral blood or BM. Although the induction therapy was not consolidated with allogeneic stem cell transplantation (allo-SCT), our patient remains in complete cytogenetic and molecular remission, on single-agent dasatinib, 3.5 years after the diagnosis with no current evidence of active extramedullary disease. This suggests that dasatinib has a role in controlling not only chronic-phase CML, but also its CNS blast crisis, especially when combined with whole-brain radiation therapy.

\section{Case Presentation}

We present the case of a 29-year-old Saudi man who presented initially in May 2012 with a 2-week history of headaches and double vision. His family noticed some bouts of confusion during the same period. He denied other neurological and systemic symptoms.

His physical examination revealed a moderately built young male in no acute distress. His vital signs were within normal limits. He had no organomegaly or evidence of skin or mucosal bleeding. His neurological examination showed an altered sensorium and signs of 


\section{Case Reports in Oncology}

right sixth nerve palsy with no meningismus. Upon presentation, his laboratory data showed normal electrolytes and liver and kidney functions. His lactate dehydrogenase measured 668 $\mathrm{U} / \mathrm{l}$, and his complete blood counts had a hemoglobin level of $17 \mathrm{~g} / \mathrm{dl}$, a white blood cell count (WBC) of $34.3 \times 10^{9} / \mathrm{l}$, and a platelet count of $853 \times 10^{9} / \mathrm{l}$. His WBC differential showed $55 \%$ neutrophils, $17 \%$ lymphocytes, $2 \%$ eosinophils, $12 \%$ bands, $6 \%$ metamyelocytes, $5 \%$ myelocytes and no promyelocytes or basophils. His peripheral blood smear showed leftshifted neutrophilia with toxic granulations together with thrombocytosis and no blasts. Computed tomography (CT) of the brain performed upon admission was within normal limits. Although retrospectively his laboratory results were consistent with chronic-phase CML, due to the absence of a prior history and due to his CNS symptoms and signs he was treated empirically for bacterial meningitis. In-patient treatment with meropenem, ampicillin and vancomycin was initiated. Unfortunately, he showed no clinical or radiological improvement. After 7 days of admission, he became agitated, and his Glasgow coma scale dropped to 8/15, necessitating a transfer to the intensive care unit.

Repeated CT of the brain performed upon transfer to the intensive care unit showed a new 4-cm left frontoparietal subdural collection and another adjacent collection within the left frontotemporal lobe measuring $1.5 \mathrm{~cm}$, causing a mass effect and shift to the right. The CT also showed a right frontoparietal subdural collection with an adjacent frontal collection, which was indicative of an infection. The patient underwent transcranial drainage of the subdural fluid, the analysis of which showed a cloudy yellowish cerebrospinal fluid (CSF) with increased protein levels but no evidence of infection by Gram stain or culture. The CSF's total WBC was 2,356 $\times 10^{9} / \mathrm{l}$ with $1 \%$ polymorphs, $3 \%$ lymphocytes and $95 \%$ blasts (fig. 1 a). Flow cytometry of the CSF showed that $65 \%$ of blast cells were positive for a cluster of differentiation markers [CCD)34, CD33, CD11b and CD71] and partially positive for CD13 and CD117, confirming CNS extramedullary myeloid blast infiltration.

Magnetic resonance imaging (MRI) of the brain showed postdrainage of the left frontoparietal subdural collection as well as diffuse meningeal thickening and enhancement involving both cerebral hemispheres, more on the left side. The interhemispheric fissure and all sulci indicated diffuse meningitis. In addition, the MRI showed a 6-mm subdural collection at the right superior temporal region. There were 2 enhancing lesions in the vestibular and abducent nerves (fig. 2).

CT of the chest, abdomen and pelvis with intravenous contrast showed multiple mediastinal, para-aortic and peripancreatic enlarged lymph nodes; the largest measured $3.4 \times 3.3$ $\mathrm{cm}$. The spleen and liver were within normal limits.

A CT-guided left para-aortic lymph node biopsy showed diffuse infiltration by myeloblasts, which was consistent with granulocytic sarcoma. The blasts were positive for CD45, CD34, CD68, CD38 and CD43 and negative for MPO, CD20, CD3, CD79a, PAX5, TdT, S100 and cytokeratin, consistent with a diagnosis of blast-phase CML (fig. 1b-f).

The patients underwent BM aspiration and biopsy, and the result was consistent with a diagnosis of chronic-phase CML. Conventional cytogenetics yielded $t(9 ; 22)$ in all examined cells; it also showed trisomy 8,14 and 21 and $\operatorname{del}(16 q)$. The BM aspirate was positive for the BCR-ABL oncogene when analyzed by fluorescence in situ hybridization and polymerase chain reaction. BM findings were consistent with chronic-phase CML with no excess blasts despite the confirmed extramedullary CNS and lymph node blast crises.

The patient was started on dasatinib $70 \mathrm{mg}$ orally twice daily together with conventional acute myelogenous leukemia induction with a cytarabine and idarubicin $(7+3)$ regimen with 16 doses of intrathecal methotrexate/cytarabine/hydrocortisone. He subsequently developed prolonged pancytopenia with multiple infections. Owing to the patient's poor 


\section{Case Reports in Oncology}

general condition, an allo-SCT was not recommended, despite the availability of a matched sibling donor. Instead, our patient was treated with $24 \mathrm{~Gy}$ of radiation therapy delivered in 12 fractions to the whole brain in the period from January 19 through March 3, 2013. Since then, the patient has continued to receive dasatinib $100 \mathrm{mg}$ orally daily, being followed by 3 monthly polymerase chain reactions for BCR-ABL oncogene from peripheral blood and annual fluorescence in situ hybridization test from BM aspirate. He remains in complete cytogenetic and molecular remission with no current evidence of active extramedullary disease according to his latest brain MRI performed 4 months prior to writing this report.

\section{Discussion}

Although CML has become an indolent illness in the majority of patients, blast crises still represent serious and often life-threatening complications that require aggressive measures to control the disease and prepare the patient for an allo-SCT when feasible. Extramedullary blast crises have traditionally been linked to poor survival [3]. However, our patient is still living with no evidence of extramedullary disease relapse.

CNS blast crisis usually presents with the clinical and radiological features of meningitis and/or encephalitis, which includes symptoms such as headache, seizures, meningismus and/or focal neurological symptoms and signs. The CSF is found positive for myeloid/lymphoid blasts, and in some cases, molecular testing of the CSF has revealed the typical BCR-ABL oncogene [8]. Our patient's initial presentation was suggestive of meningitis with cranial nerve palsy and change in mental status. Although we have not confirmed the presence of the BCR-ABL oncogene in his CSF, our patient had a significant amount of myeloid blasts in his CSF and the presence of BCR-ABL oncogene was evident in his peripheral blood and BM.

Our case presented with the typical features of subacute meningitis with toxic granulation in a left-shifted moderate neutrophilia and in the absence of splenomegaly; longstanding CML seemed unlikely owing to the absence of prior history. All of these factors made early diagnosis a challenge. Careful examination of the CSF for atypical cells/blasts is crucial in such cases, as early appropriate therapy can prevent permanent neurological deficits.

Traditional therapy for CNS involvement by Philadelphia-positive leukemia composed of radiotherapy, intrathecal and high-dose systemic chemotherapy is unsatisfactory and leads to short-term responses [17]. In managing such cases, an appropriate selection of a tyrosine kinase inhibitor (TKI) with a higher blood-brain barrier penetration to be added to systemic and CNS-directed therapy is crucial. Dasatinib is a second-generation TKI that has a 325-fold greater potency in inhibiting BCR-ABL oncogene compared to imatinib in vitro [18]. We selected to use dasatinib in our patient instead of first-generation TKI imatinib as it has been proven to be able to penetrate the blood-brain barrier and to have a significant longstanding response for CNS Philadelphia-positive leukemias [19]. There is an urgent need for novel strategies to treat such complicated cases, perhaps by incorporating therapies used for primary CNS cancer, such as high-dose methotrexate or temozolomide. Dasatinib may have an important role in managing CNS CML blast crises, especially when combined with wholebrain radiation therapy. 


\section{Statement of Ethics}

No patient's identifier is included in this report; however, the patient consented prior to the writing of this paper.

\section{Disclosure Statement}

All authors declare to have no conflict of interest.

\section{References}

1 Rowley JD: Letter: a new consistent chromosomal abnormality in chronic myelogenous leukaemia identified by quinacrine fluorescence and Giemsa staining. Nature 1973;243:290-293.

-2 Kantarjan HM, Keating MJ, Talpaz M: Chronic myelogenous leukemia in blast crisis: analysis of 242 patients. Am J Med 1987;83:445-454.

-3 Canellos G: Clinical characteristics of the blast phase of chronic granulocytic leukemia. Hematol Oncol Clin North Am 1990;4:359-376.

4 Specchia G, Palumbo G, Pastore D, Mininni D, Mestice A, Liso V: Extramedullary blast crises in chronic myeloid leukemia. Leuk Res 1996;20:905-908.

5 Matsuda M, Morita Y, Shimasa T, Miyatake J, Hirase C, Tanaka M, Tasumi Y, Maeda Y, Kanamaru A: Extramedullary blast crisis derived from 2 different clones in the central nervous system and neck during complete cytogenetic remission of chronic myelogenous leukemia treated with imatinib mesylate. Int J Hematol 2005;81:307-309.

-6 Ganessan K, Goel R, Kumar K, Bakhshi S: Biphenotypic extramedullary blast crisis as a presenting manifestation of Philadelphia chromosome-positive CML in a child. Pediatr Hematol Oncol 2007;24:195-198.

7 Al-Rayes HM, Al-Shaikh AA, Halim MA, Al-Qurashi FS, Al-Jurf MM: Leukemic synovitis as a presentation of myelomonocytic blast crisis of chronic myeloid leukemia. Saudi Med J 2001;22:808-811.

8 Jin GN, Zou P, Chen WX, Ding ZY, Zhou H: Fluorescent in situ hybridization diagnosis of extramedullary nodal blast crisis. Diagn Cytopathol 2013;41:253-256.

-9 Shune L, Cayci Z, Rogosheske J, Brunstein C, Ustun C: Extramedullary blastic crisis in abdominal lymph nodes in a patient with chronic myelogenous leukemia on imatinib. Leuk Res 2012;36:e131-e132.

10 Yu HH, Lu MY, Lin DT, Lin KH, Tang JL, Jou ST: Pathological fracture as a manifestation of extramedullary blastic crisis in chronic myelogenous leukemia: report of one case. Acta Paediatr Taiwan 2006;47:150-154.

-11 Rajappa S, Uppin SG, Raghunadharao D, Rao IS, Surath A: Isolated central nervous system blast crisis in chronic myeloid leukemia. Hematol Oncol 2004;22:179-181.

12 Radhika N, Minakshi M, Rajesh M, Manas BR, Kumar MD: Central nervous system blast crisis in chronic myeloid leukemia on imatinib mesylate therapy: report of two cases. Indian J Hematol Blood Transfus 2011;27:51-54.

$13 \mathrm{Kim} \mathrm{Hj}$, Jung CW, Kim K: Isolated blast crisis in CNS in a patient with chronic myelogenous leukemia maintaining major cytogenetic response after imatinib. J Clin Oncol 2006;24:4028-4029.

14 Bornhauser M, Jenke A, Freiberg-Richter J, Radke J, Schuler US, Mohr B, et al: CNS blast crisis of chronic myelogenous leukemia in a patient with major cytogenetic response in bone marrow associated with low levels of imatinib mesylate and its $\mathrm{N}$-desmethylated metabolite in cerebral spinal fluid. Ann Hematol 2004;83:401-402.

15 Wolff NC, Richardson JA, Egorin M, Ilaria RL Jr: The CNS is a sanctuary for leukemic cells in mice receiving imatinib mesylate for Bcr/Abl-induced leukemia. Blood 2003;101:5010-5013.

-16 Isobe Y, Sugimoto K, Masuda A, Hamano Y, Oshimi K: Central nervous system is a sanctuary site for chronic myelogenous leukemia treated with imatinib mesylate. Intern Med 2009;39:408-418.

17 Cortes J: Central nervous system involvement in adult acute lymphoblastic leukemia. Hematol Oncol Clin North Am 2001;15:145-162.

-18 O'Hare T, Walters DK, Stoffregen EP, Jia T, Manley PW, Mestan J, et al: In vitro activity of Bcr-Abl inhibitors AMN107 and BMS-354825 against clinically relevant imatinib-resistant Abl kinase domain mutants. Cancer Res 2005;65:4500-4505. 


\section{Case Reports in Oncology}

\begin{tabular}{l|l}
\hline Case Rep Oncol 2016;9:415-421 \\
\hline $10.1159 / 000447711$ & $\begin{array}{l}\text { C } 2016 \text { The Author(s). Published by S. Karger AG, Basel } \\
\text { www.karger.com/cro }\end{array}$ \\
\hline
\end{tabular}

Abuelgasim et al: An Atypical Initial Presentation of Chronic Myeloid Leukemia with Central Nervous System and Lymph Node Blast Crises

19 Porkka K, Koskenvesa P, Lundan T, Rimpilainen J, Mustjoki S, Smykla R, et al: Dasatinib crosses the blood-brain barrier and is an efficient the therapy for central nervous system Philadelphia chromosome-positive leukemia. Blood 2008;112:1005-1012.
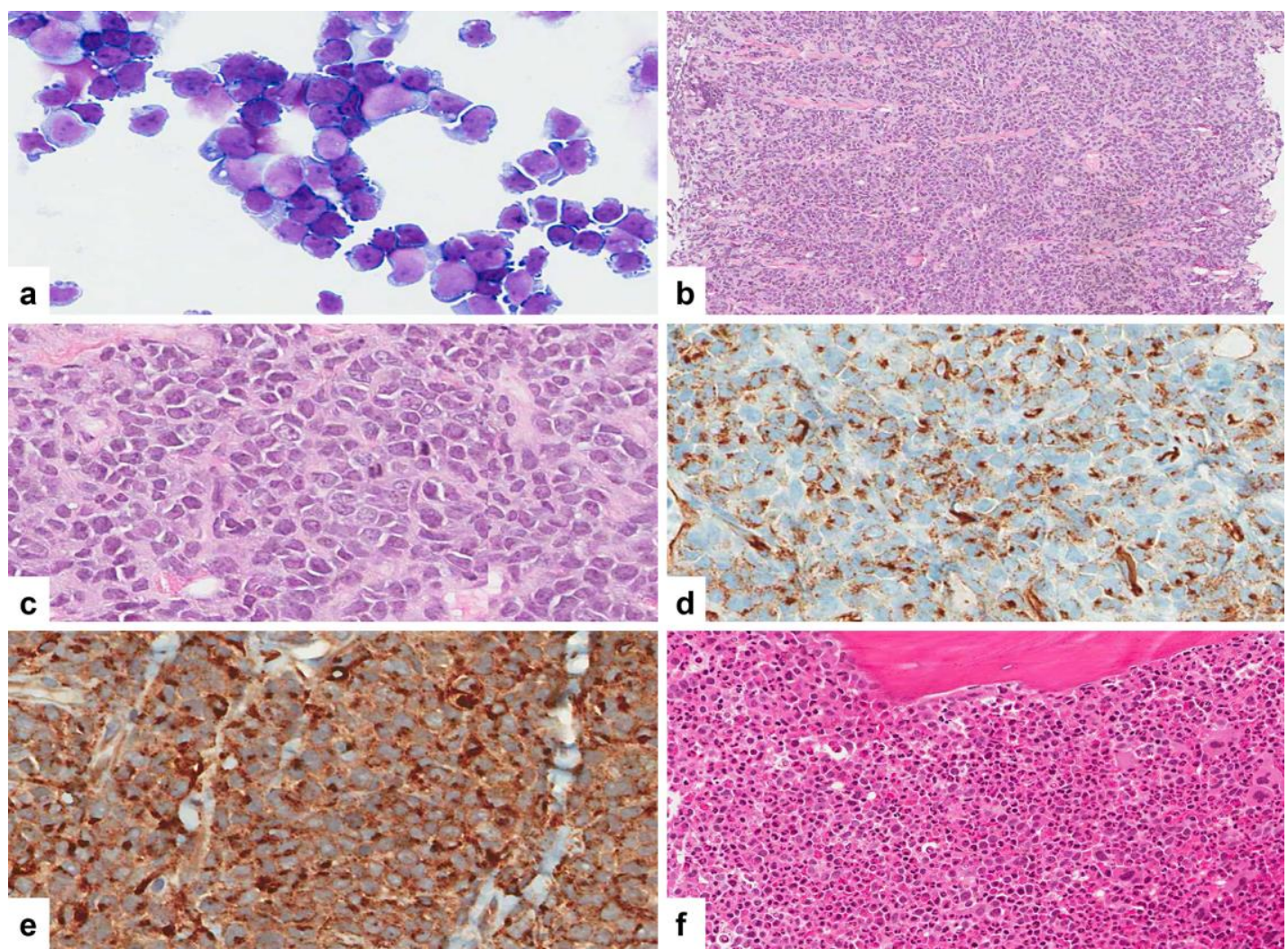

Fig. 1. Findings of CSF, lymph nodes showing excess blasts and BM biopsy with no excess blasts at the time of diagnosis. a The CSF is hypercellular and shows many blasts $(\times 400$, Giemsa stain). $\mathbf{b}$ The lymph node needle core stained with hematoxylin and eosin shows sheets of large atypical cells (blasts) ( $\times 100)$. c Highpower view of the lymph node needle core stained with hematoxylin and eosin shows large irregular nuclei, visible nucleoli and frequent mitotic figures $(\times 400)$. $\mathbf{d}$ The blasts in the lymph node needle core are positive for CD34. e The blasts in the lymph node needle core are positive for CD68 immunostains. $\mathbf{f}$ The BM trephine biopsy stained with hematoxylin and eosin showing features of CML with no increased blasts $(\times 100)$. 


\section{Case Reports in Oncology}
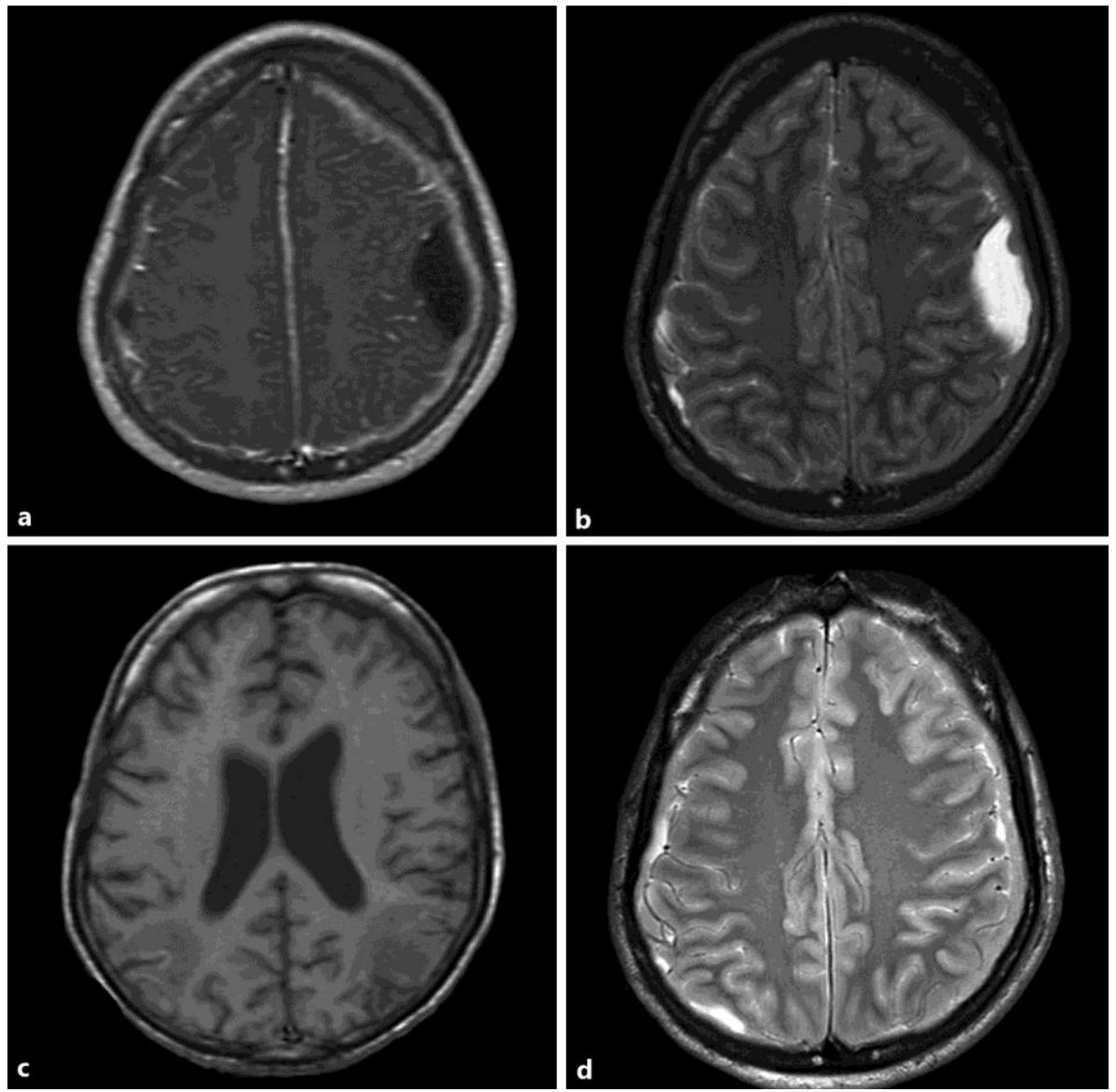

Fig. 2. MRI of the brain before and after the therapy. T1 imaging (a) and T2 imaging (b) before the therapy: diffuse meningeal thickening and enhancement involving both cerebral hemispheres (more on the left side). A 6-mm subdural collection at the right superior temporal region. T1 imaging (c) and T2 imaging (d) after the therapy: complete resolution of the previously enhanced areas. 